\title{
Consensus Modelling on Interval-Valued Fuzzy Preference Relations with Normal Distribution
}

\author{
Lihong Wang, Zaiwu Gong*, Ning Zhang \\ Collaborative Innovation Center on Forecast and Evaluation of Meteorological Disasters, School of Management \\ Science and Engineering, Nanjing University of Information Science and Technology, \\ No.219 Ningliu Road, Nanjing, Jiangsu, 210044, China \\ E-mail: zwgong26@163.com
}

Received 19 February 2017

Accepted 5 February 2018

\begin{abstract}
This paper investigates the consensus decision making problem of the interval-valued fuzzy preference relation with distribution characteristics. The proposed group consensus decision making model is constructed by considering the scenarios in which the DMs are respectively equally and non-equally weighted and the DM's preferences are randomly distributed. The goal is to find the minimum deviation between an ideal DM and all individual DMs. Accordingly, the objective function is the maximum consensus with a certain probability. The interactive process simulates the DM's uncertainty judgment information more effectively. The Pareto optimization solution derived using a genetic algorithm and Monte Carlo approach is closer to reality. In the process of solving the model in this study, the essence of the Monte Carlo simulation method is an interactive process involving decision information. Therefore, this study provides a reference for the framework and optimization algorithm of the interactive decision support system.
\end{abstract}

Keywords: Group decision making (GDM), interval-valued fuzzy preference relation, normal distribution, genetic algorithm (GA), group consensus

\section{Introduction}

In many studies on preference relations (PRs) ${ }^{1,2,3,4}$, the decision makers (DMs) or experts must evaluate the options and provide their preferences through pairwise comparisons to construct their judgment matrices. The earliest work on PRs mostly involved use of the reciprocal $\mathrm{PR}^{2}$ and the complementary fuzzy PR (FPR) ${ }^{1}$, both of which are represented by crisp numbers. Owing to the complexity of group decision making (GDM), DMs are more likely to express their preferences using uncertainty judgments, such as the interval-valued fuzzy preference relation (IVFPR) ${ }^{5,6}$ for each pair of alternatives to formulate a more effective expression.
The advantage of the IVFPR is its intuitiveness. Specifically, the IVFPR elements are represented by real interval-valued numbers, where the interval's lower limit is interpreted as the most conservative preference and the upper limit indicates the most optimistic preference. The IVFPR has become a useful structure in obtaining the priorities of alternatives and evaluating the consistency and consensus degrees of individuals to enable a more reasonable decision making process. The core achievements of IVFPR are primarily consistency and group consensus.

A brief summary of the research into consistency is as follows: Consistency is an important property of PR that is used to estimate whether the DM employs a mathematical logic when they give their judgment

\footnotetext{
* Corresponding author.
} 
information. It also serves as the foundation for ranking alternatives and integrating group preference information, and is a precondition of GDM. A lack of consistency may lead to inconsistent decisions ${ }^{7}$. Since Saaty $^{2}$ and Tanino $^{3}$ established the definitions of multiplicative consistency and additive consistency, respectively, research on consistency has served as the theoretical foundation for all types of uncertainty PRs. Khalid and Beg introduced an upper bound condition to address incomplete IVFPR ${ }^{8}$. Moreover, Alonso et al. presented a procedure that can maintain experts' consistency levels in estimating missing preference values when handling pairwise comparisons and heterogeneous information ${ }^{9}$. The procedure to estimate missing values can be applied to incomplete fuzzy, multiplicative, interval-valued, and linguistic PRs. In addition, Meng et al. ${ }^{10}$ focused on multiplicative consistency research with IVFPRs, and they have achieved significant results.

A brief summary of group consensus research is as follows: The consensus-reaching process is necessary to obtain a final solution with a certain level of agreement among the DMs. Group consensus is a consistent measurement of all DMs' PRs that handle various issues, such as how to solve the contradiction of DMs' PRs, how to assimilate multivariate PRs, and how to aggregate multi-granular linguistic preference information into reliable collective PRs. For example, Gong et al. proposed two consensus models with interval preference opinions and gave their economic interpretation ${ }^{11}$. Pérez-Fernández et al. presented a twofold GDM problem based on finitely generated sets and finite interval-valued hesitant fuzzy preference relations (IVHFPRs) ${ }^{12}$. This twofold GDM problem engenders a novel perspective on the decision making problem in that several experts and criteria can be simultaneously considered. Meanwhile, Liu et al. proposed a method based on a sentiment analysis technique and intuitionistic fuzzy set theory to rank products based on online reviews ${ }^{13}$. Tooranloo and sadat Ayatollah proposed a model for a failure mode and effects analysis based on an intuitionistic fuzzy approach ${ }^{14}$. Furthermore, Büyüközkan and Güleryüz proposed a combined intuitionistic fuzzy GDM model $^{15}$ comprising an intuitionistic fuzzy analytic hierarchy process ${ }^{16}$ and an intuitionistic fuzzy "technique for order preference by similarity to ideal solution" (TOPSIS) approach for effectively evaluating product development partners. Wang et al. developed a fractional programming model based on a TOPSIS method to determine a relative closeness interval, whereby attribute weights are independently determined for each alternative ${ }^{17}$. Zhao et al. developed three interactive intuitionistic fuzzy methods to address multilevel programming problems in hierarchy expert and intelligent systems ${ }^{18}$.
Despite the above efforts, disadvantages exist in the mathematical operations of both priority modelling and group consensus modelling with IVFPRs. This is especially the case for multiplication and division of interval numbers, which can cause excessive amplification or reduction of decision information, leading to the distortion of decision results. Nevertheless, high-level relationships exist between interval numbers and random distributions, such as the normal distribution and uniform distribution. Thus, we can approximately represent the interval number with a random variable that obeys normal distribution using mapping relations. Moreover, using the random distribution can more effectively express the range of DM judgments during a pairwise comparison of any alternatives. For example, the average value and variance can be easily obtained in this process. Considering the random distribution characteristic of DM judgments in GDM, the group consensus degree can also be measured based on probability programming $^{19}$. In GDM, a unanimous consensus cannot always be achieved; nonetheless, a widespread consensus is easy to realize. At the same time, the condition cannot be achieved with a full percentage of the group; however, it is acceptable for the condition to be realized with a certain probability (e.g. 95\% probability). This scenario is closer to practical situations in which a group consensus is achieved with a certain probability.

In GDM, decision making must be completed before the realization of random variables (the individual's preference). However, if the probability that these conditions can be satisfied is no less than a certain index, these problems can be solved with probabilistic constraint programming. This stochastic programming method was proposed in 1959 by Charnes and Cooper ${ }^{20}$ and is renowned for realizing optimization under a certain probability. For some special situations, chancerestricted programming can be equally transformed into determined mathematical programming. However, for some complex chance constrained problems, a random simulation based genetic algorithm $(\mathrm{GA})^{21}$ is a useful approach in solving chance constrained, general chance constrained, and chance constrained multi-objective programming problems. Hong et al. proposed the use of a gradient-based Monte Carlo method to solve the sequence of convex approximations ${ }^{22}$. Olson and $\mathrm{Wu}$ used chance constrained models in financial planning ${ }^{23}$. Charnes and Cooper presented a method that splits the problem into two non-linear (or linear) programming parts $^{20}$. The first problem is determining the optimal probability distributions; the second is approximating the optimal distributions as closely as possible by decision rules of a certain form. 
Many theoretical results have been achieved in probability-restricted programming methods that address problems with random distribution variables. We use this method to deal with group consensus optimization problems with randomly distributed preferences. In addition, by employing a GA, we derive the optimum values of the objective function of the consensus optimization problem and obtain a satisfactory solution of variables.

The remainder of this paper is organized as follows. In Section 2, we define FPR, IVFPR, and consistent FPR. We then construct an IVFPR consensus optimization model based on the minimum deviation of preference between the ideal DM and all DMs. In Section 3, we construct four consensus models of IVFPR with random distributions of individual judgment. In Section 4, a numerical example is presented, and the Pareto optimization solution is simulated using the Monte Carlo and GA. The paper is concluded in Section 5.

\section{Preliminaries}

For a GDM problem, let $X=\left\{x_{1}, x_{2}, \cdots, x_{n}\right\}$ be a finite set of alternatives and let $D=\left\{d_{1}, d_{2}, \cdots, d_{m}\right\}$ be the set of DMs. We denote $N=\{1,2, \cdots, n\} \quad$ and $M=\{1,2, \cdots, m\}$.

Definition of $\mathbf{F P R}^{3}$. According to their respective experiences and knowledge, the DMs make pairwise judgments on any two alternatives, $x_{i}$ and $x_{j}(i, j \in N)$, over the set of $X$ regarding a decision criterion. An FPR $R$ on a set of alternatives $X$ is represented by a complementary matrix:

$$
R=\left(\begin{array}{cccc}
r_{11} & r_{12} & \cdots & r_{1 n} \\
r_{21} & r_{22} & \cdots & r_{2 n} \\
\vdots & \vdots & \ddots & \vdots \\
r_{n 1} & r_{n 2} & \cdots & r_{n n}
\end{array}\right)
$$

where $r_{i j}+r_{j i}=1, r_{i i}=0.5,0 \leq r_{i j} \leq 1$, and $r_{i j}$ represents a crisp preference degree of the alternative $x_{i}$ over $x_{j}, i, j \in N$. Specifically, $r_{i j}=0$ indicates that $x_{i}$ is absolutely preferred to $x_{j}, r_{i j}=0.5$ indicates no difference between $x_{i}$ and $x_{j}, r_{i j}>0.5$ indicates that $x_{i}$ is preferred over $x_{j}$, and $r_{i j}=1$ indicates that $x_{i}$ is absolutely not preferred over $x_{j}$.

Considering the complexity of the decision making environment, the DMs tend to provide an interval value to ensure a more effective judgment expression of any two alternatives, $x_{i}$ and $x_{j}(i, j \in N)$, over the set $X$ with respect to a decision criterion. The lower limit of the interval-value indicates the expert's most conservative judgment, while the upper limit indicates the expert's most optimistic judgment.

Definition of IVFPR ${ }^{24}$. An IVFPR is represented by an interval-valued fuzzy preference matrix:

$$
\bar{R}=\left(\bar{r}_{i j}\right)_{n \times n}=\left(\begin{array}{cccc}
{\left[r_{11 l}, r_{11 u}\right]} & {\left[r_{12 l}, r_{12 u}\right]} & \cdots & {\left[r_{1 n l}, r_{1 n u}\right]} \\
{\left[r_{21 l}, r_{21 u}\right]} & {\left[r_{22 l}, r_{22 u}\right]} & \cdots & {\left[r_{2 n l}, r_{2 n u}\right]} \\
\vdots & \vdots & \ddots & \vdots \\
{\left[r_{n 1 l}, r_{n 1 u}\right]} & {\left[r_{n 2 l}, r_{n 2 u}\right]} & \cdots & {\left[r_{n n l}, r_{n n u}\right]}
\end{array}\right)
$$

where $r_{i j l}+r_{j i u}=1, \quad r_{i j u}+r_{j i l}=1, \quad 0 \leq r_{i j l} \leq r_{i j u} \leq 1$, $r_{i i l}=r_{i i u}=0.5$, and $\bar{r}_{i j}$ is a continuous interval value, which indicates that the preference degree or intensity of alternative $x_{i}$ over that of $x_{j}(i, j \in N)$ is between $r_{i j l}$ and $r_{i j u}$.

Definition of consistent $\mathbf{F P R}^{25}$. Let $R=\left(r_{i j}\right)_{n \times n}$ be an FPR where the following equation is satisfied: $r_{i j}+r_{j k}=r_{i k}+0.5, i, j, k \in N, i \neq j \neq k$. Then, we call $R$ a consistent FPR.

Relationship between the Interval Number and the Normally Distributed Variables ${ }^{26}$. In real decision making, although the experts can give their interval judgment value for any pairwise comparison of alternatives, they cannot give a more accurate value. They can only determine the approximate probability distribution of their judgment, such as a normal distribution. Considering that the interval itself originates from numerical results of fuzzy judgment or random sampling, only the range of interval numbers (the upper and lower bounds) is known. However, it is difficult to determine the real value of the interval number; i.e. the interval number can be handled as a fuzzy number and also regarded as a random variable. Therefore, it is reasonable to use a random variable instead of a particular interval number. In the absence of a priori knowledge, these random variables may be normally distributed, uniformly distributed, chi-squared distributed, etc. We consider the advantageous property of normal distribution. For a random variable $\xi$ of normal distribution $N\left(\mu, \sigma^{2}\right)$, according to the $3 \sigma$ principle, the probability of falling in the interval $[\mu-3 \sigma, \mu+3 \sigma]$ is $99.73 \%$. According to the $3 \sigma$ law,

$$
\left[a^{-}, a^{+}\right]=[\mu-3 \sigma, \mu+3 \sigma] \quad, \quad \mu=\frac{a^{-}+a^{+}}{2} \quad, \quad \text { and }
$$


$\sigma=\frac{a^{+}-a^{-}}{6}$, so we can assign the normally distributed random variables to all intervals ${ }^{14}$. For example, by the use of the $3 \sigma$ law, the interval number $I=[0.6,0.9]$ can be approximately transformed into a random variable $\xi$ which satisfies $\xi \sim N\left(0.7500,0.0500^{2}\right)$.

\section{Consensus Model with IVFPR}

In GDM, let $\bar{R}^{k}=\left(\bar{r}_{i j}^{k}\right)_{n \times n}$ be the IVFPR of the k-th DM, where $\bar{r}_{i j}^{k}=\left[r_{i j l}^{k}, r_{i j u}^{k}\right]$ is interpreted as the membership of the k-th DM's preference degree of the alternative $x_{i}$ over $x_{j}$ that satisfies $r_{i j l}^{k}+r_{j i u}^{k}=1, \quad r_{i j u}^{k}+r_{j i l}^{k}=1$, $0 \leq r_{i j l}^{k} \leq r_{i j u}^{k} \leq 1, r_{i i l}^{k}=r_{i i u}^{k}=0.5$ for all $i, j \in N$. Here $r_{i j l}^{k}$ indicates the most conservative degree of the k-th DM's preference of the alternative $x_{i}$ over $x_{j}$, and $r_{i j u}^{k}$ indicates the most optimistic degree of the alternative $x_{i}$ over $x_{j}$.

Suppose there is an ideal DM (moderator), of whom the FPR is $R^{*}=\left(r_{i j}^{*}\right)_{n \times n}$ (we suppose that the moderator's ideal judgment is FPR instead of IVFPR), which satisfies $r_{i j}^{*}+r_{j i}^{*}=1,0 \leq r_{i j}^{*} \leq 1, \quad r_{i i}^{*}=0.5$, $i, j \in N$. Moreover, assume that the FPR of the ideal $\mathrm{DM}$ is unknown. The minimum deviation optimization model between the moderator's IVFPR and the IVFPR of all individual DMs is introduced as ${ }^{27}$

$$
\begin{aligned}
U & =\left[\sum_{k=1}^{m} \sum_{i=1}^{n} \sum_{j=1}^{n}\left|\bar{r}_{i j}^{k}-\bar{r}_{i j}^{*}\right|^{p}\right]^{\frac{1}{p}} \\
& =\left[\sum_{k=1}^{m} \sum_{i=1}^{n} \sum_{j=1}^{n}\left|r_{i j l}^{k}-r_{i j}^{*}\right|^{p}\right]^{\frac{1}{p}}+\left[\sum_{k=1}^{m} \sum_{i=1}^{n} \sum_{j=1}^{n}\left|r_{i j u}^{k}-r_{i j}^{*}\right|^{p}\right]^{\frac{1}{p}}
\end{aligned}
$$

where $1 \leq p<\infty$. In this model, the smaller the deviation is, the better it is; i.e. the smaller the value of $U$ is, the greater the consensus is. Further, assume that $\omega_{k}$ is the weight of the $\mathrm{k}$-th individual DM which satisfies both $0 \leq \omega_{k} \leq 1$ and $\sum_{k=1}^{m} \omega_{k}=1$. Then, a minimum deviation optimization model between the moderator's IVFPR and the IVFPR of all individual DMs with weights is introduced as ${ }^{27}$

$$
\begin{aligned}
& U=\left[\sum_{k=1}^{m} \sum_{i=1}^{n} \sum_{j=1}^{n} \omega_{k}\left|\bar{r}_{i j}^{k}-\bar{r}_{i j}^{*}\right|^{p}\right]^{\frac{1}{p}} \\
& \text { s.t. } 0 \leq r_{i j}^{*} \leq 1, i, j \in N
\end{aligned}
$$

where

$U=\left[\sum_{k=1}^{m} \sum_{i=1}^{n} \sum_{j=1}^{n} \omega_{k}\left|r_{i j l}^{k}-r_{i j}^{*}\right|^{p}\right]^{\frac{1}{p}}+\left[\sum_{k=1}^{m} \sum_{i=1}^{n} \sum_{j=1}^{n} \omega_{k}\left|r_{i j u}^{k}-r_{i j}^{*}\right|^{p}\right]^{\frac{1}{p}}$, and $1 \leq p<\infty$.

This indicates the weighted average of the deviation between the ideal judgment value $\bar{r}_{i j}^{*}$ and the judgments of $\mathrm{m} \mathrm{DMs} \bar{r}_{i j}^{k}, p$ represents different consensus choice rules $^{28}$. The smaller the value of $U$, the greater the consensus between the moderator and the individual DMs.

In group consensus model (1) the upper and lower limits of the interval-valued judgement are simultaneously considered; however, considering whole intervals is not possible. Considering the disadvantages of interval operations, it is difficult to show the actual situation of GDM by the ideal FPR derived from model (1). In this paper, the interval-valued judgment is approximately replaced by a normally distributed variable. Consequently, the uncertainty of the decision result is reduced and the authenticity of the decision is improved.

\section{Consensus Modelling of IVFPR with Distribution Characteristics}

In actual decision making, DMs provide their judgments by pairwise comparisons of alternatives. Nevertheless, the crisp numbers of their preferences are difficult to determine. We can only determine the approximate probability distribution of a DM's judgment, such as by a normal distribution. In this section, we assume that the interval-valued judgment $\bar{r}_{i j}^{k}$ of the $\mathrm{k}$-th $\mathrm{DM}$ of the comparison between alternatives $x_{i}$ and $x_{j}$ is normally distributed, that is, $\bar{r}_{i j}^{k} \sim N\left(u_{i j}^{k},\left(\sigma_{i j}^{k}\right)^{2}\right)$. Meanwhile, we suppose there is an ideal DM in GDM, and the DM's FPR is $R^{*}=\left(r_{i j}^{*}\right)_{n \times n}$, where $0 \leq r_{i j}^{*} \leq 1$.

In reality, although uniform $(100 \%)$ consensus of GDM is difficult to achieve, the moderator also desires to realise a cardinal agreement of the DMs to a certain degree. In this paper, consensus is interpreted in terms of a certain probability. For example, to achieve a $90 \%$ probability of group consensus or an assurance of 0.9 , 
four consensus models of IVFPR with distribution characteristics will be constructed:

(1) Suppose that all DMs are equally weighted and the judgment values of their preferences are approximately normally distributed. Then, the deviation between the moderator's judgment and the judgments of all DMs can be obtained. The probability of an event occurring in which the deviation is no more than threshold $\varepsilon$ is no less than the probability level $\alpha$, and the objective function is the minimum value of this deviation. Then, group consensus model (2) of IVFPR with entries satisfying a normal distribution can be built as follows:

$$
\begin{aligned}
& \min \varepsilon \\
& \text { s.t. }\left\{\begin{array}{l}
P_{r}\left\{\left[\sum_{k=1}^{m} \sum_{i=1}^{n} \sum_{j=1}^{n}\left|\bar{r}_{i j}^{k}-r_{i j}^{*}\right|^{p}\right]^{\frac{1}{p}} \leq \varepsilon\right\} \geq \alpha \\
\bar{r}_{i j}^{k} \sim N\left(u_{i j}^{k},\left(\sigma_{i j}^{k}\right)^{2}\right) \\
0 \leq r_{i j}^{*} \leq 1 \\
i, j \in N, k \in M
\end{array}\right.
\end{aligned}
$$

(2) Suppose that all DMs are non-equally weighted and the judgment values of their preferences are approximately normally distributed. Then, we can modify the above model to account for the different DM weights as follows:

$\min \varepsilon$

$$
\text { s.t. }\left\{\begin{array}{l}
P_{r}\left\{\left[\sum_{k=1}^{m} \sum_{i=1}^{n} \sum_{j=1}^{n} \omega_{k}\left|\bar{r}_{i j}^{k}-r_{i j}^{*}\right|^{p}\right]^{\frac{1}{p}} \leq \varepsilon\right\} \geq \alpha \\
\bar{r}_{i j}^{k} \sim N\left(u_{i j}^{k},\left(\sigma_{i j}^{k}\right)^{2}\right) \\
0 \leq r_{i j}^{*} \leq 1 \\
i, j \in N, k \in M
\end{array}\right.
$$

(3) Suppose that all DMs are equally weighted and the judgment values of their preferences are approximately normally distributed. Then, the deviation between the moderator's judgment and the judgments of all DMs can be obtained. Considering the consistency property of the ideal DM's judgment, the probability of an event occurring in which the deviation is no more than threshold $\varepsilon$ is no less than the probability level $\alpha$, and the objective function is the minimum value of this deviation. Then, group consensus model (4) of IVFPR with entries satisfying a normal distribution can be built as follows:

$$
\begin{aligned}
& \min \varepsilon \\
& \text { s.t. }\left\{\begin{array}{l}
P_{r}\left\{\left[\sum_{k=1}^{m} \sum_{i=1}^{n} \sum_{j=1}^{n}\left|\bar{r}_{i j}^{k}-r_{i j}^{*}\right|^{p}\right]^{\frac{1}{p}} \leq \varepsilon\right\} \geq \alpha \\
\bar{r}_{i j}^{k} \sim N\left(u_{i j}^{k},\left(\sigma_{i j}^{k}\right)^{2}\right) \\
\left|r_{i j}^{*}+r_{j k}^{*}-\left(r_{i k}^{*}+0.5\right)\right| \leq \eta \\
0 \leq r_{i j}^{*} \leq 1, i, j \in N, k \in M
\end{array}\right.
\end{aligned}
$$

In models (4) and (5), the constraint $\left|r_{i j}^{*}+r_{j k}^{*}-\left(r_{i k}^{*}+0.5\right)\right| \leq \eta, \quad i, j \in N \quad$ indicates that the judgment of the ideal DM satisfies approximate consistency. Moreover, the smaller the value of $\eta$ is, the better the consistency is.

(4) Suppose that all DMs are non-equally weighted and the judgment values of their preferences are approximately normally distributed. Then, we can modify model (4) to account for the different DM weights as follows:

$$
\begin{aligned}
& \min \varepsilon \\
& \text { s.t. }\left\{\begin{array}{l}
P_{r}\left\{\left[\sum_{k=1}^{m} \sum_{i=1}^{n} \sum_{j=1}^{n} \omega_{k}\left|\bar{r}_{i j}^{k}-r_{i j}^{*}\right|^{p}\right]^{\frac{1}{p}} \leq \varepsilon\right\} \geq \alpha \\
\bar{r}_{i j}^{k} \sim N\left(u_{i j}^{k},\left(\sigma_{i j}^{k}\right)^{2}\right) \\
\left|r_{i j}^{*}+r_{j k}^{*}-\left(r_{i k}^{*}+0.5\right)\right| \leq \eta \\
0 \leq r_{i j}^{*} \leq 1, i, j \in N, k \in M
\end{array}\right.
\end{aligned}
$$

Remarks: In models (2) to (5), the value of $p$ satisfies $1 \leq p<\infty$. The smaller the value of objective function $\varepsilon$, the greater the consensus. In these four models, the distribution of the k-th DM's judgment can also approximately be replaced by a uniform distribution, i.e. $\bar{r}_{i j}^{k} \sim U\left(r_{i j l}^{k}, r_{i j u}^{k}\right)$, which can be solved by similar approaches. For ease of calculation, in the following numerical examples, we only consider $p=1$.

\section{Numerical Examples}

For this GDM example, we assume there are three individual DMs $d_{1}, d_{2}$, and $d_{3}$ and an ideal moderator 
$d^{*}$. Their IVFPRs on alternatives $A_{1}, A_{2}, A_{3}$, and $A_{4}$ are $\bar{R}^{1}, \bar{R}^{2}, \bar{R}^{3}$, and $R^{*}$, respectively.

$$
\begin{aligned}
& \bar{R}^{\prime}=\left(\begin{array}{l}
{[0.5,0.5][0.2,0.4][0.3,0.4][0.6,0.9]} \\
{[0.6,0.8][0.5,0.5][0.6,0.7][0.6,0.7]} \\
{[0.6,0.7][0.3,0.4][0.5,0.5][0.7,0.7]} \\
{[0.1,0.4][0.3,0.4][0.3,0.3][0.5,0.5]}
\end{array}\right), \\
& \bar{R}^{\prime}=\left(\begin{array}{l}
{[0.5,0.5][0.2,0.4][0.3,0.6][0.5,0.7]} \\
{[0.6,0.8][0.5,0.5][0.7,0.9][0.6,0.8]} \\
{[0.4,0.7][0.1,0.3][0.5,0.5][0.7,0.8]} \\
{[0.3,0.5][0.2,0.4][0.2,0.3][0.5,0.5]}
\end{array}\right), \\
& \bar{R}^{\prime}=\left(\begin{array}{l}
{[0.5,0.5][0.6,0.7][0.8,0.9][0.7,0.8]} \\
{[0.3,0.4][0.5,0.5][0.6,0.7][0.6,0.7]} \\
{[0.1,0.2][0.3,0.4][0.5,0.5][0.5,0.6]} \\
{[0.2 .0 .3][0.3,0.4][0.4,0.5][0.5,0.5]}
\end{array}\right) .
\end{aligned}
$$

Based on the relationship between the interval number and the normally distributed variables proposed in Section $2, \bar{R}^{1}, \bar{R}^{2}$ and $\bar{R}^{3}$ can be approximately transformed into the following respective PRs with a normally distributed judgment:

$$
N^{1} \sim
$$

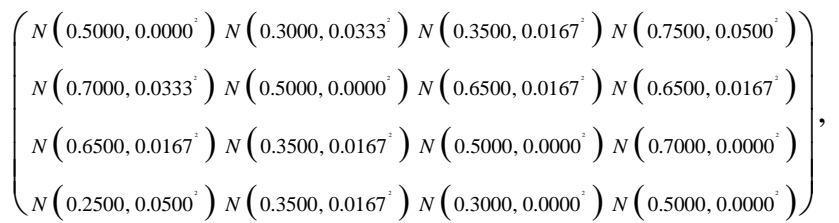
$N^{2} \sim$

$\left(\begin{array}{l}N\left(0.5000,0.0000^{3}\right) N\left(0.3000,0.0333^{3}\right) N\left(0.4500,0.0500^{3}\right) N\left(0.6000,0.0333^{3}\right) \\ N\left(0.7000,0.0333^{\prime}\right) N\left(0.5000,0.0000^{\prime}\right) N\left(0.8000,0.0333^{3}\right) N\left(0.7000,0.0333^{3}\right) \\ N\left(0.5500,0.0500^{3}\right) N\left(0.2000,0.0333^{3}\right) N\left(0.5000,0.0000^{3}\right) N\left(0.7500,0.0167^{\prime}\right) \\ N\left(0.4000,0.0333^{\prime}\right) N\left(0.3000,0.0333^{\prime}\right) N\left(0.2500,0.0167^{\prime}\right) N\left(0.5000,0.0000^{\prime}\right)\end{array}\right)$, $N^{3} \sim$

$\left(\begin{array}{l}N\left(0.5000,0.0000^{\prime}\right) N\left(0.6500,0.0167^{\prime}\right) N\left(0.8500,0.0167^{\prime}\right) N\left(0.7500,0.0167^{\prime}\right) \\ N\left(0.3500,0.0167^{\prime}\right) N\left(0.5000,0.0000^{\prime}\right) N\left(0.6500,0.0167^{\prime}\right) N\left(0.6500,0.0167^{\prime}\right) \\ N\left(0.1500,0.0167^{\prime}\right) N\left(0.3500,0.0167^{\prime}\right) N\left(0.5000,0.0000^{\prime}\right) N\left(0.5500,0.0167^{\prime}\right) \\ N\left(0.2500,0.0167^{\prime}\right) N\left(0.3500,0.0167^{\prime}\right) N\left(0.4500,0.0167^{\prime}\right) N\left(0.5000,0.0000^{\prime}\right)\end{array}\right)$.

The moderator's FPR $R^{*}$ will be derived by the following numerical models.

Case 1: Assume the three DMs are equally weighted. Based on model (2), we construct group consensus model (6) with normally distributed PRs:

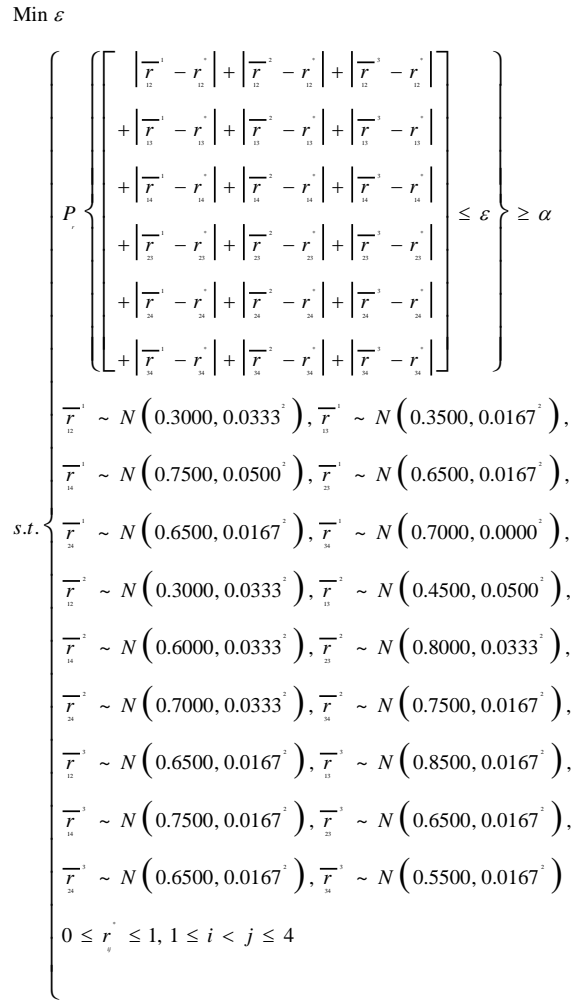

Case 2: Assume the three DMs are non-equally weighted. The corresponding weights are $0.2,0.3$, and 0.5 , respectively. Based on model (3), we construct group consensus model (7) with normally distributed PRs: 


$$
\begin{aligned}
& \text { Min } \varepsilon
\end{aligned}
$$

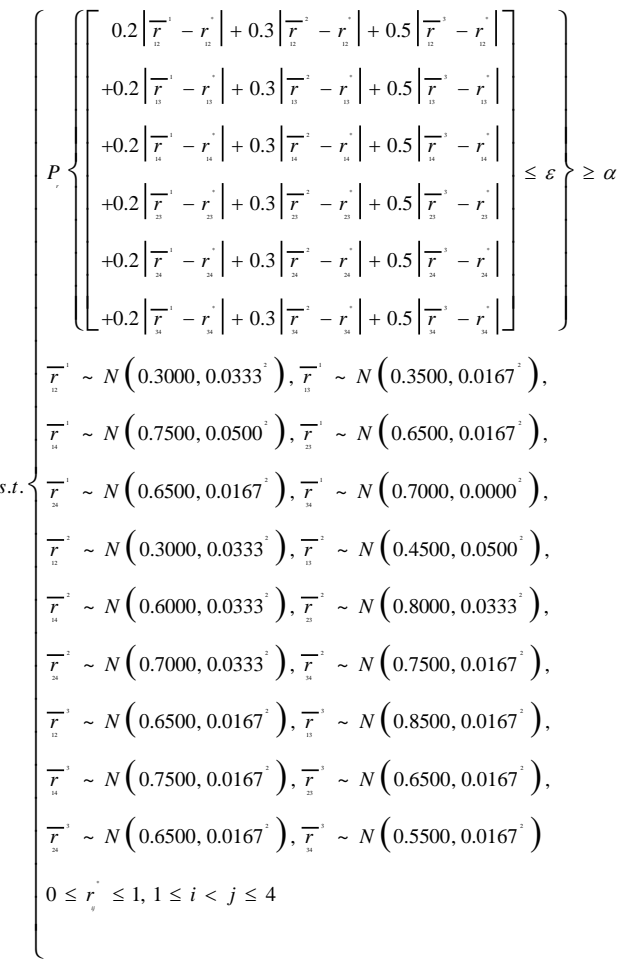

Case 3: Assume the three DMs are equally weighted. Considering the consistency property of the ideal DM's judgment information, based on model (4), we construct group consensus model (8) with normally distributed PRs:

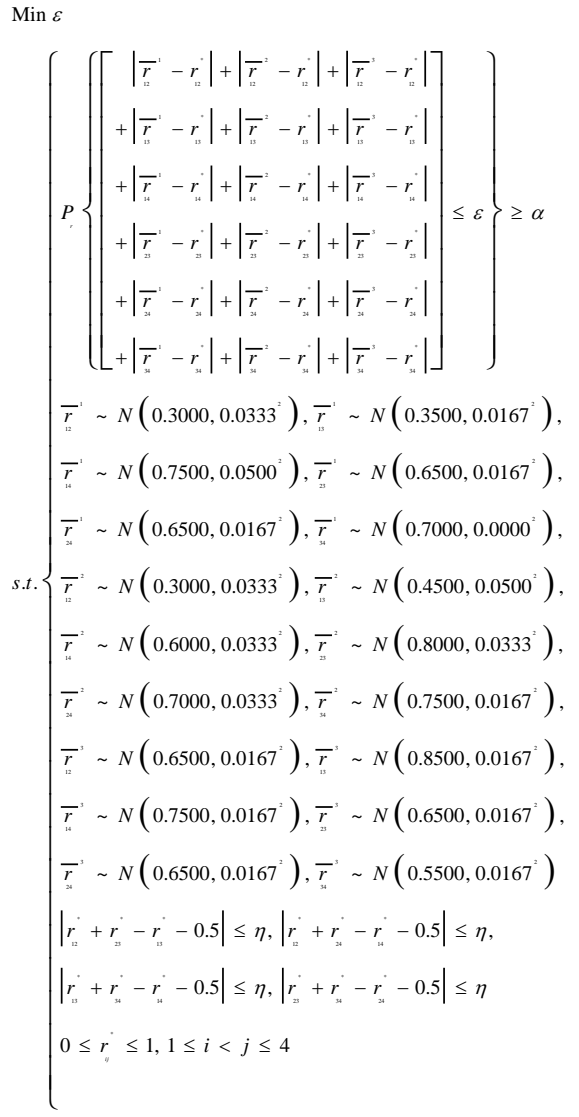

It should be noted that, because complete consistency of the DM's judgment is difficult to achieve, in models (7) and (8), the smaller the value of $\eta$, the better the consistency. In these models we suppose that $\eta=0.05$.

Case 4: Assume the three DMs are non-equally weighted, and the corresponding weights are $0.2,0.3$, and 0.5 respectively. Based on model (5), we construct group consensus model (9) with normally distributed PRs: 


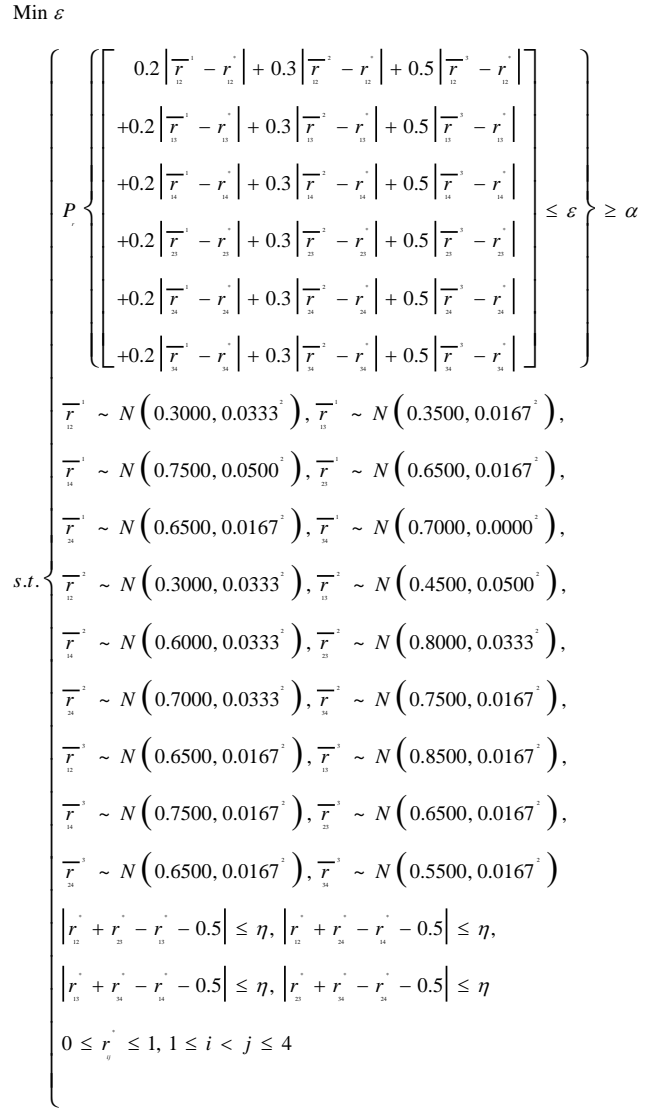

In the above numerical examples, we assume that the probability levels are $\alpha=0.90,0.95,0.98,0.99$, and 1.00 respectively. Using the GA, the Pareto optimization solutions of models (6) to (9) are shown in Table 1.

Table 1 Pareto optimization solutions of models (6) to (9)

\begin{tabular}{|c|c|c|c|c|c|c|c|c|}
\hline \multirow[b]{2}{*}{$\begin{array}{l}\text { Numerical } \\
\text { examples }\end{array}$} & \multirow{2}{*}{$\begin{array}{c}\text { Probability } \\
\text { level } \\
\alpha\end{array}$} & \multirow[b]{2}{*}{$r_{12}^{*}$} & \multicolumn{5}{|c|}{ Elements of the ideal DM's preference relation } & \multirow[b]{2}{*}{$\operatorname{Min} \varepsilon$} \\
\hline & & & $r_{13}^{*}$ & $r_{14}^{*}$ & $r_{23}^{*}$ & $r_{24}^{*}$ & $r_{34}^{*}$ & \\
\hline \multirow[t]{5}{*}{ (6) } & 0.90 & 0.6523 & 0.8515 & 0.0640 & 0.9142 & 0.6647 & 0.4291 & 2.2872 \\
\hline & 0.95 & 0.6560 & 0.5572 & 0.3177 & 0.6678 & 0.8737 & 0.9543 & 2.3220 \\
\hline & 0.98 & 0.6573 & 0.8244 & 0.4075 & 0.9976 & 0.2116 & 0.5218 & 2.3613 \\
\hline & 0.99 & 0.6583 & 0.0013 & 0.3477 & 0.9198 & 0.6640 & 0.9147 & 2.3805 \\
\hline & 1.00 & 0.6560 & 0.0952 & 0.7496 & 0.2573 & 0.9882 & 0.5567 & 2.4326 \\
\hline \multirow[t]{5}{*}{ (7) } & 0.90 & 0.6534 & 0.3840 & 0.3209 & 0.1905 & 0.1586 & 0.9942 & 0.6904 \\
\hline & 0.95 & 0.6563 & 0.0970 & 0.7184 & 0.6011 & 0.2913 & 0.1461 & 0.7011 \\
\hline & 0.98 & 0.6549 & 0.1816 & 0.2954 & 0.1166 & 0.9723 & 0.3575 & 0.7124 \\
\hline & 0.99 & 0.6498 & 0.9762 & 0.2494 & 0.9408 & 0.7395 & 0.2707 & 0.7201 \\
\hline & 1.00 & 0.6515 & 0.9807 & 0.3772 & 0.1706 & 0.8133 & 0.4794 & 0.7312 \\
\hline \multirow[t]{3}{*}{ (8) } & 0.90 & 0.6542 & 0.7582 & 0.9932 & 0.5711 & 0.7968 & 0.7045 & 2.3074 \\
\hline & 0.95 & 0.6581 & 0.7611 & 0.8931 & 0.6180 & 0.7719 & 0.6425 & 2.3537 \\
\hline & 0.98 & 0.6547 & 0.1267 & 0.4511 & 0.0049 & 0.3197 & 0.8431 & 2.3833 \\
\hline
\end{tabular}

Table 1 (Continued)

\begin{tabular}{ccccccccc}
\hline \multirow{2}{*}{$\begin{array}{c}\text { Numerical } \\
\text { examples }\end{array}$} & $\begin{array}{c}\text { Probability } \\
\text { level }\end{array}$ & $r_{12}^{*}$ & $r_{13}^{*}$ & $r_{14}^{*}$ & $r_{23}^{*}$ & $r_{24}^{*}$ & $r_{34}^{*}$ & Min $\varepsilon$ \\
& $\alpha$ & & & & & \\
& 0.99 & 0.6631 & 0.6348 & 0.8621 & 0.4529 & 0.7347 & 0.7729 & 2.4295 \\
& 1.00 & 0.6407 & 0.5232 & 0.4388 & 0.3751 & 0.3339 & 0.4586 & 2.5395 \\
& 0.90 & 0.6504 & 0.7888 & 0.9250 & 0.6664 & 0.7851 & 0.5963 & 0.6964 \\
& 0.95 & 0.6505 & 0.4660 & 0.5820 & 0.3284 & 0.4601 & 0.6291 & 0.7099 \\
& 0.98 & 0.6557 & 0.2951 & 0.3097 & 0.1882 & 0.1487 & 0.4688 & 0.7206 \\
& 0.99 & 0.6617 & 0.5350 & 0.7909 & 0.3952 & 0.6463 & 0.7494 & 0.7319 \\
& 1.00 & 0.6579 & 0.8270 & 0.4665 & 0.6399 & 0.3088 & 0.1280 & 0.7612 \\
\hline
\end{tabular}

Based on the data in Table 1, we can obtain the FPR of the ideal DM in four cases. In particular, the ideal judgment values obtained by models (8) and (9) approximately satisfy the consistency constraint. In the solving process of models (6) to (9), the interval values were transformed into approximately equivalent random variables with normal distributions. Moreover, the Pareto optimization solutions from the stochastic simulation and GA were obtained, which avoids the limitations of operations over intervals. It should also be noted that the priorities of the ideal FPRs can be obtained by conventional ranking models; see References 29 and 30.

\section{Conclusions}

In GDM, IVFPR has been widely used. The typical method is to construct the optimization model based on the FPR consistency and then derive the optimization solution of GDM with the IVFPR. However, this method has many disadvantages, especially in applying operations to intervals, where the upper and lower limits must be simultaneously considered. To some extent, the multiplication and division of intervals can cause excessive amplification or reduction of decision information, which leads to the distortion of decision results. In this paper, we strived to transform the interval-valued preference of DMs into random variables which obey normal distributions, and to construct four consensus models of IVFPR with distribution characteristics.

Two constraints were proposed for the above models. The first constraint is the deviation, $\varepsilon$, between the ideal DM's FPR and the IVFPR of all individual DMs with normal variations that are no more than a certain limit under probability level $\alpha$. In other words, the probability of the event occurring in which the deviation is no more than threshold $\varepsilon$ exceeds the probability level of $\alpha$. The second constraint is the judgment of the ideal DM satisfying the consistency property. Per 
the objective function, the smaller the value of $\varepsilon$, the better the consensus of GDM. We can then obtain the Pareto optimization solution, which is more suitable for practice by the usage of the Monte Carlo technique and GA.

The innovative elements of this study are summarized as follows. Firstly, based on the relationship between the interval number and the random variable with normal distribution, the random variable preference with normal distribution is used to approximately replace the interval-valued preference. Secondly, the upper and lower bounds of the interval preference do not need to be considered in the decision making of group consensus modelling. Rather, the distribution characteristics of the DM's preference are directly considered. This approach can efficiently avoid the distortion of decision information during operations on intervals. Lastly, a GA is used to simulate the intervalvalued GDM process with a normal distribution to obtain a more practical optimization solution. In the process of solving the model in this study, the essence of the Monte Carlo simulation method is an interactive process of decision information. Therefore, this study provides a reference for the framework and optimization algorithm of the interactive decision support system.

\section{Acknowledgements}

The work in this paper was supported by the National Natural Science Foundation of China (71571104, 71171115, and 70901043); Qing Lan Project; the Six Talent Peaks Project in Jiangsu Province (2014-JY-014); the Project Funded by the Priority Academic Program Development of Jiangsu Higher Education Institutions and the Natural Science Foundation of Jiangsu, China (grant No. BK20141481); and the fifth issue of the "333 Project" research projects funded project (BRA2017456).

\section{Appendix}

The details of the proposed probabilistic programming method based on GA are as follows:

Step 1: randomly generate initial population between all variables' stated ranges based on GA;

Step 2: randomly generate some variables obeying a certain distribution;

Step 3: combine initial population with random variables and evaluate whether all generated variables satisfy the constraints. If yes, then go Step 5; if no, then go Step 4;
Step 4: through selective reproduction, crossing over, and mutation, go into generation cycle and go to Step 2;

Step 5: collect all feasible populations and obtain the optimal solution.

\section{References}

1. S. A. Orlovsky, Decision making with a fuzzy preference relation, Fuzzy Set. and Syst. 1(3), (1978) 155-167.

2. T. L. Saaty, Modeling unstructured decision problems - the theory of analytical hierarchies, Mathematics and Computers in Simulation. 20(3), (1978) 147-158.

3. T. Tanino, Fuzzy preference orderings in group decision making, Fuzzy Set. and Syst. 12(2), (1984) 117-131.

4. T. L. Saaty, The analytic hierarchy process, McGraw-Hill, New York. (1980) (p. 287).

5. T. L. Saaty and L. G. Vargas, Uncertainty and rank order in the analytic hierarchy process, Eur. J. Oper. Res. 32(1), (1987) 107-117.

6. H. Ishibuchi and H. Tanaka, Multiobjective programming in optimization of the interval objective function, Eur. J. Oper. Res. 48(2), (1990) 219-225.

7. F. Chiclana, E. Herrera-Viedma, S. Alonso and F. Herrera, Cardinal Consistency of Reciprocal Preference Relations: A Characterization of Multiplicative Transitivity, IEEE T. Fuzzy Syst. 17(1), (2009) 14-23.

8. A. Khalid and I. Beg, Incomplete interval valued fuzzy preference relations, Inform. Sciences $\mathbf{3 4 8}$ (2016) 15-24

9. S. Alonso, F. Chiclana, F. Herrera, E. HerreraViedma, J. Alcalá-Fdez, and C. Porcel, A consistency-based procedure to estimate missing pairwise preference values, Int. J. Intell. Syst. 23 (2) (2008) 155-175.

10. F. Meng, C. Tan and X. Chen, Multiplicative consistency analysis for interval fuzzy preference relations: A comparative study, Omega 68 (2017) 17-38.

11. Gong, Z., Xu, X., Zhang, H., Ozturk, U. A., Herrera-Viedma, E. and $\mathrm{Xu}, \mathrm{C}$. (2015). The consensus models with interval preference opinions and their economic interpretation. Omega, 55 81-90.

12. R. Pérez-Fernández, P. Alonso, H. Bustince, I. Díaz and S. Montes, Applications of finite intervalvalued hesitant fuzzy preference relations in group decision making, Inform. Sciences 326 (2016) 89101. 
13. Y. Liu, J. W. Bi and Z. P. Fan, Ranking products through online reviews: A method based on sentiment analysis technique and intuitionistic fuzzy set theory, Inform. Fusion 36 (2017) 149-161.

14. H. S. Tooranloo and A. sadat Ayatollah, A model for failure mode and effects analysis based on intuitionistic fuzzy approach, Appl. Soft Comput. 49 (2016) 238-247.

15. G. Büyüközkan and S. Güleryüz, A new integrated intuitionistic fuzzy group decision making approach for product development partner selection, Comput. Ind. Eng. 102 (2016) 383-395.

16. T. L. Saaty, Analytic hierarchy process, John Wiley Sons Ltd. (1980).

17. Z. Wang, K. W. Li and J. Xu, A mathematical programming approach to multi-attribute decision making with interval-valued intuitionistic fuzzy assessment information, Expert Syst. Appl. 38 (10) (2011) 12462-12469.

18. X. Zhao, Y. Zheng and Z. Wan, Interactive intuitionistic fuzzy methods for multilevel programming problems, Expert Syst. Appl. 72 (2016) 258-268.

19. N. Kushmerick, S. Hanks and D. S. Weld, An algorithm for probabilistic planning. Artif. Intell. 76 (1-2) (1995) 239-286.

20. A. Charnes and W. W. Cooper, Chance-constrained programming, Manage. Sci. 6 (1) (1959) 73-79.

21. J. H. Holland, Adaptation in natural and artificial systems. MIT Press. (1992).

22. L. J. Hong, Y. Yang and L. Zhang, Sequential convex approximations to joint chance constrained programs: A Monte Carlo approach. Oper. Res. 59 (3) (2011) 617-630.

23. D. L. Olson and D. D. Wu, Chance Constrained Models, in Enterprise Risk Management Models, Springer-Verlag GmbH, Germany, (2017) 89-103.

24. F. Herrera, L. Martínez and P. J. Sánchez, Managing non-homogeneous information in group decision making, Eur. J. Oper. Res. 166 (1) (2005) $115-132$.

25. E. Herrera-Viedma, F. Herrera, F. Chiclana and M. Luque, Some issues on consistency of fuzzy preference relations, Eur. J. Oper. Res. 154 (1) (2004) 98-109.

26. M. Chen, S. G. Wang, P. P. Wang and X. Ye, A new equivalent transformation for interval inequality constraints of interval linear programming, Fuzzy Optim. Decis. Ma. 15 (2) (2016) 155-175.

27. González-Pachón, J. and Romero, C. Bentham, Marx and Rawls ethical principles: In search for a compromise. Omega 62 (2016) 47-51.

28. Tan, X., Gong, Z., Huang, M. and Wang, Z. J. Selecting Cooking Methods to Decrease Persistent Organic Pollutant Concentrations in Food of Animal Origin Using a Consensus Decision making Model. Int. J. Env. Res. Pub. He. 14(2) (2017) 187.

29. Z. W. Gong, Least-square method to priority of the fuzzy preference relations with incomplete information, Int. J. Approx. Reason. 47 (2) (2008) 258-264.

30. Z. Xu and Q. Da, A least deviation method to obtain a priority vector of a fuzzy preference relation, Eur. J. Oper. Res. 164 (1) (2005) 206-216. 